Full Proceeding Paper

\title{
BIDIRECTIONAL RECURRENT NEURAL NETWORK FOR IMPROVING MULTISPECTRAL IMAGE DENOISING
}

\author{
ANKUSH RAI, JAGADEESH KANNAN R \\ School of Computing Science and Engineering, VIT University, Chennai, Tamil Nadu, India. Email: ankushressci@gmail.com
}

Received: 28 December 2016, Revised and Accepted: 10 May 2017

\begin{abstract}
Objective: While procuring images from satellite the multispectral images (MSIs) are often prone to noises. Finding a good mathematical description of the learning-based denoising model is a difficult research question and many different researches accounted in the literature.

Methods: Many have attempted its use with the application of neural network as a sparse learned dictionary of noisy patches. Furthermore, this approach allows several algorithm to optimize itself for the given task at hand by using machine learning algorithm. In this study, we present an improved method for learning-based denoising of MSI images. Recurrent neural network used in this study helps in speeding up the computational operability and denoising performance by over $85-95 \%$.
\end{abstract}

Results and Conclusion: The results from this study not only provides sparsity of corollary of homogenous blocks but also gives show superiority over other methods using cited in the literature.

Keywords: Recurrent neural network, Multispectral imaging, Denoising algorithm.

(C) 2017 The Authors. Published by Innovare Academic Sciences Pvt Ltd. This is an open access article under the CC BY license (http://creativecommons. org/licenses/by/4. 0/) DOI: http://dx.doi.org/10.22159/ajpcr.2017.v10s1.19678

\section{INTRODUCTION}

The filtering based approach for image denoising heavily relies of general arguments which varies drastically as it either holds for all sorts of multispectral images (MSI) or in other cases even for the digital signals also [1,2]. For illustration, this technique holds the assumption that the pixels values will not change from one pixel to another, and thereby the neighboring pixels can be thought as similar samples to the pixels that are in the current iteration to get denoised. Algorithms for this technique include:

- Mean filtering

- Median filtering

- Bilateral filtering

- Anisotropic diusion.

Such methods does not require any sort of pre-processing technique for performing computational operation of denoising and thus speed up the whole process in general. Although, due to its generality, the obtained results from such techniques are inferior to the task specific algorithms.

There are also some methods which rely on global statistics of the image which also makes some general assumptions about the images. Therefore, in such types of denoising algorithm at least some part of computation are done or the training of denoising pixels are achieved to extract the general statistics of the image. For illustration, there could have the requirement of for a dictionary of clean imagery patches, and thus the function for neighboring pixels are based on Markov random fields $[3,4]$.

The Algorithms which are based on or should we say which exploits the statistics of the natural image, i.e., its scarcity of pixels in Fourier space or on the basis of wavelet give empirically better performance since such empirical studies of the MSI require to perform computation prerequisite of formulating the denoised pattern in this approach. The methods of this approach include:

- Wavelet or Dictionary-based methods which relies on decompositionbased methods, such as $[5,6]$

- $\quad$ Learned filter based methods [7,8].
Since, in this approach, the output is already pushed toward the realistic model of the imagery data. Thus, it performs better than the previously discussed general filtering techniques with low computational time since the global statistics requires to be computed only once.

This method heavily relies on the statistics found in the image itself. The algorithms compute on the basis of per patches which include the classes of non-local means [9]. BM3D algorithm is one of the states of the art methods used for the application. BM3D algorithm is applicable for wavelet coefficient shrinking of the image patches which is based on general statistics of the MSI [10].

These methods have been shown to work among the best in practice and have the additional advantage that no extra image statistics are required during runtime. However, these methods tend to have long run times since a lot of local evaluations in input images have to be performed.

In this category of denoising algorithms, the attempts have been made to compositely use the above two methods in combination with each other which comprise both internal and external statistics of the image. Thus, outperforming its predecessors. This has been shown in some of the few previous studies to achieve comparable results over the large number of patches related with the task of MSI denoising with a super resolution $[11,12]$.

It is clear that there requires a certain assumption associated with the processing of the prior signal information which must be used to enable the denoising algorithm for effective operation with the prior supplement of the knowledge into it. Now, for the achievement of the same, there is prior statistical information that can be prefetched to the denoising algorithm using the variety of ways developed using the combination of both statistical information and machine learning algorithm $[13,14]$

Here, the use of the term prior information can be of different meaning based on its usage type. At once it can refer to the scarcity of the wavelet 


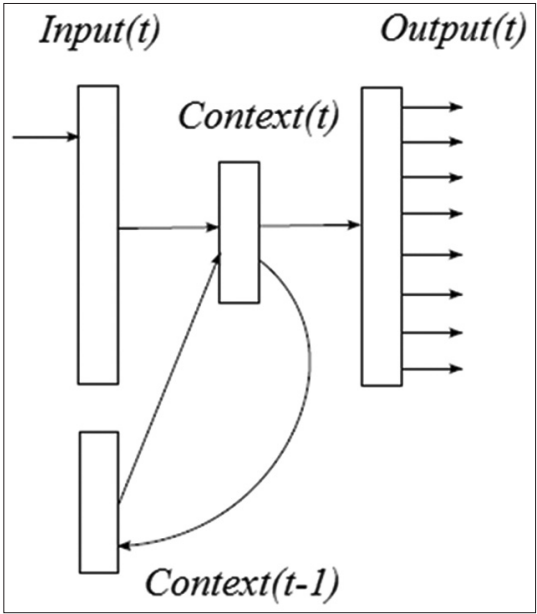

Fig. 1: Illustration of simple recurrent neural network coefficients, and at other, it refers to the probability based distribution for certain pixel value combination of small imagery patches. This type of approach can be used in multitude of image restoration tasks and poses a likelihood for better results.

Although finding a good mathematical description of the learning based denoising model is a difficult research question and many different researches accounted in the literature [15]. Many have attempted its use with the application of neural network as a sparse learned dictionary of noisy patches [16]. Furthermore, this approach allows several algorithm to optimize itself for the given task at hand by using machine learning algorithm. Thus, the same model can be used for solving different task and in different conditions [17]. That's the reason why more of the studies are based on developing an automated algorithm for multispectral denoising using recurrent neural network (RNN).

Although, in the past, there are several algorithm has been proposed to resolve the image denoising of multi-spectral images to find the approximate estimate of the noisy free signals from the recorded noisy

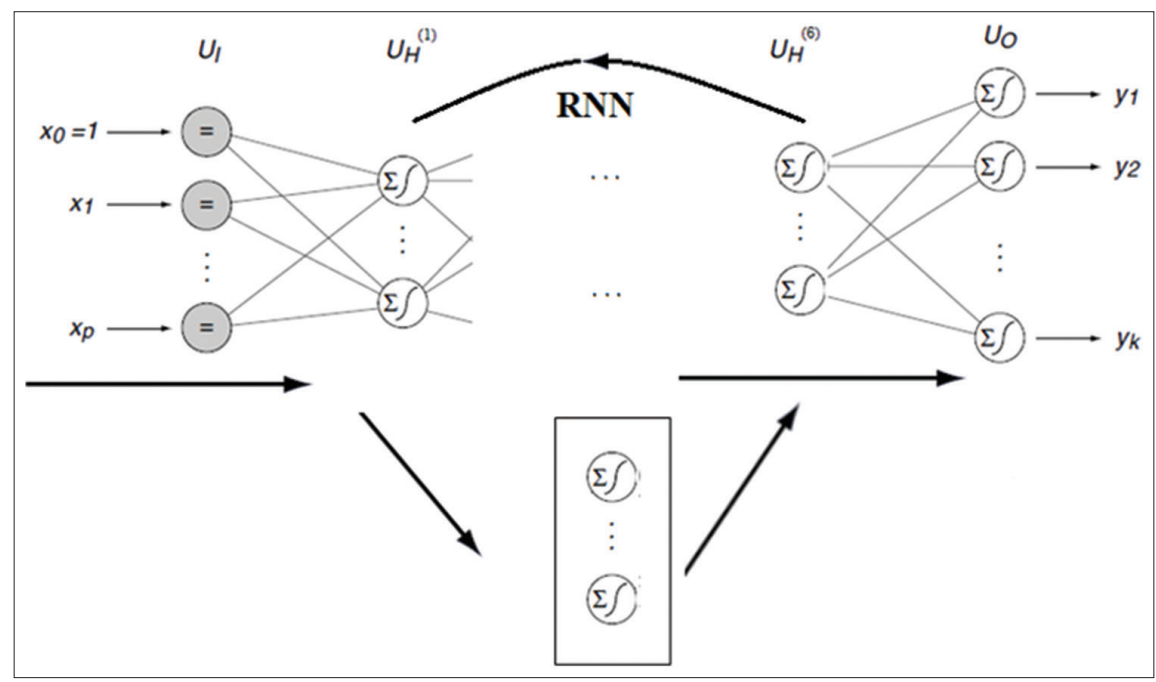

Fig. 2: Training mechanism of the MLPs

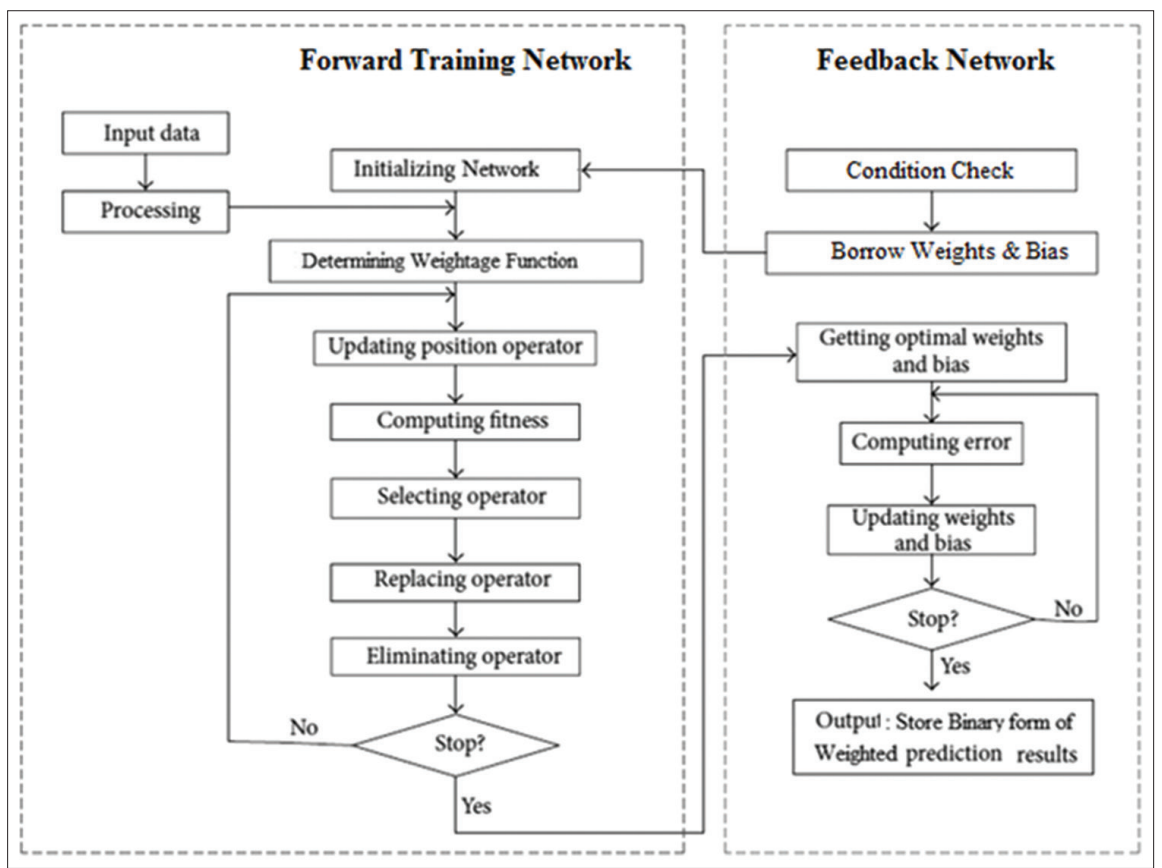

Fig. 3: Flowchart for the training of recurrent neural network 


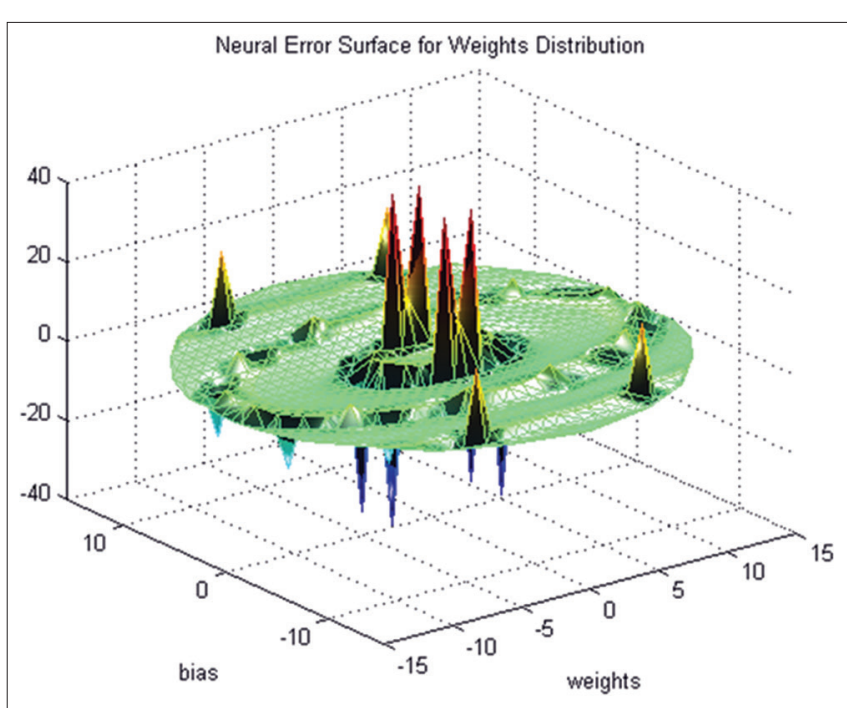

Fig. 4: Neural error surface of recurrent neural network image. Many such attempts works under a general assumption; thus leading the process to a pavement involving heavy chunk of processing with the additional requisite to manually employ thresholding techniques for denoising, which indeed is a daunting task. Further, this eliminates the mandatory elimination of some of the essential data in sacrifice to 4extract the noise free signal. The goal of this study is to give an automated denoising algorithm for the MSI effectively with that of the past attempted methods cited below.

\section{METHODOLOGY: IMPLEMENTATION OF DEMIXED PRINCIPAL COMPONENT ANALYSIS (DPCA) WITH RNN}

We have investigated RNNs which were earlier proposed by Bengio et al. $[14,16]$. This RNN approach had proved its exceptionality in various other scenarios of modeling of sequential data. The investigation of Goodman shows that the model (Fig. 1) is highly successful when a grouped model is considered that of the single one while including the class based model [15]. In later studies of Schwenk has showed in his studies that the RNN-based models give desirable improvements in context of recognition systems like that of speech recognition [17]. Since, our algorithm on DPCA proves quite beneficial thus, we implemented it with RNN to increase its effectiveness $[18,19]$.

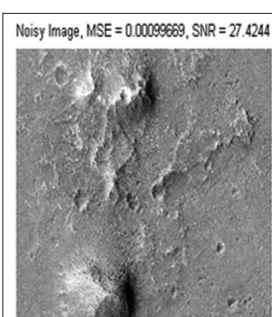

Noisy Image, MSE $=0.000000072$, SNR $=20.9817$

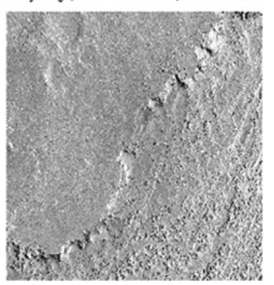

Noisy Image, MSE $=0.001001$, SNR $=23608$ I

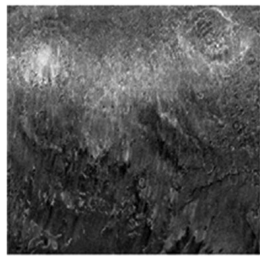

Noisy Image, MSE $=0.0009949$, SNR $=24.3548$

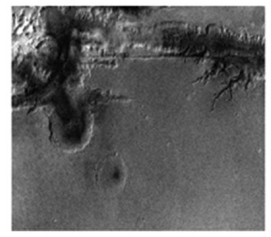

Noisy Image, MSE $=0.00099766$, SNR $=26.9998$

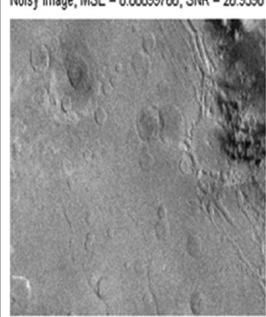

DPCA

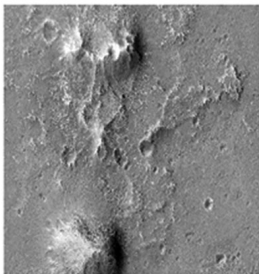

OPCA

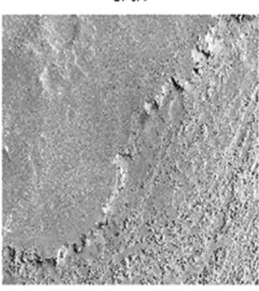

DPCA

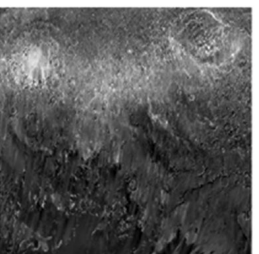

DPCA

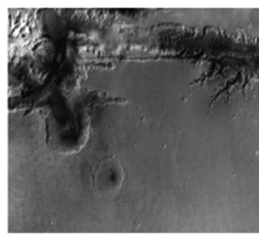

DPCA

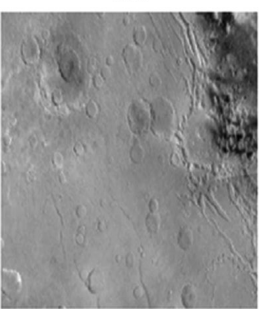

Denoised Image DPCA + RNN, MSE $=00010087$, SNR $=252572$

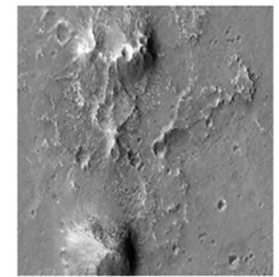

Denoised Image DPCA + RNN, MSE $=0.003711$, SNR $=23.8989$

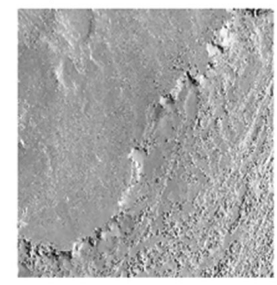

Denoised Image DPCA + RNN, MSE $=0.0012405$, SNR $=23,419$

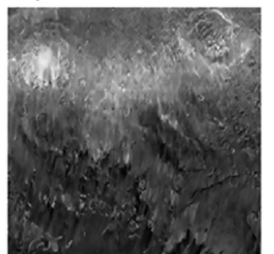

Denoised Image DPCA + RNN, MSE $=0.00052144$, SNR $=28.8323$

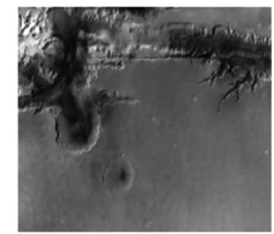

Denoised Image DPCA + RNN, MSE $=0.000056566$, SNR $=32.2523$

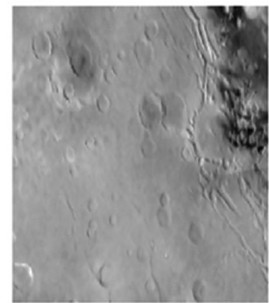

Fig. 5: Output results of denoising using the presented method. 
The standard RNN is formalized as follows: Given a sequence of input vectors $\left(X_{1}, X_{2}, X_{3}, \ldots, X_{n}\right)$, the RNN computes a sequence of hidden states $\left(\mathrm{H}_{1}, \mathrm{H}_{2}, \mathrm{H}_{3}, \ldots, \mathrm{H}_{\mathrm{n}}\right)$, and a sequence of outputs $\left(\mathrm{O}_{1}, \mathrm{O}_{2}, \mathrm{O}_{3}, \ldots, \mathrm{O}_{\mathrm{n}}\right)$, by iterating the following equations

for $\mathrm{t}=1$ to $\mathrm{n}$ :

$\mathrm{H}_{\mathrm{n}}=\tanh \left(\mathrm{w}_{\mathrm{HX}} \mathrm{X}_{\mathrm{N}}+\mathrm{w}_{\mathrm{HH}} \mathrm{H}_{\mathrm{N}-1}+\mathrm{B}_{\mathrm{N}}\right)$

$\mathrm{O}_{\mathrm{N}}=\mathrm{w}_{\mathrm{OH}} \mathrm{H}_{\mathrm{N}}+\mathrm{B}_{\mathrm{O}}$

In these equations, $\mathrm{w}_{\mathrm{HX}}$ is the input-to-hidden weight matrix, $\mathrm{w}_{\mathrm{HH}}$ is the hidden-to-hidden (or recurrent) weight matrix, $\mathrm{w}_{\mathrm{OH}}$ is the hiddento-output weight matrix, and the vectors $\mathrm{B}_{\mathrm{N}}$ and $\mathrm{B}_{0}$ are the biases. The expression replaces the inputs received from feedback loops with a special initial bias vector checked for nonlinearity while ensuring that the training is done coordinate wise.

To denoise several images, we decompose a given noisy image into overlapping patches and denoise each patch $\mathrm{x}$ separately. The workflow process of the presented algorithm is shown in Figs. 2 and 3 above. The denoised image is obtained by placing the denoised patches $f(x)$ at the locations of their noisy counterparts, then averaging on the overlapping regions. We found that we could improve results slightly by weighting the denoised patches with a Gaussian window. In addition, instead of using all possible overlapping patches (stride size 1, i.e., patch offset 1 ), we found that results were almost equally good by using every third sliding-window patch (stride size 3), while decreasing computation time by a factor of 9 . Using a stride size of 3 , we were able to denoise images of size $512 \times 419$ pixels in approximately quater of a minute on a modern CPU, which is faster than previously cited papers [18].

The gradients of the RNN is easy to compute through time but in reality the relationship between the parameters and the dynamics of the RNN is highly unstable which cause the gradient to descent ineffective. Thus, the variance we set for the gradients to make learning very stable is denoted by $\| \Delta_{\mathrm{w}} \mathrm{f}(\mathrm{x})_{\mathrm{n}}^{\mathrm{m}}$. As gradient descent was the our main DPCA algorithm used for training RNNs at the time, these results significantly reduces the empirical difficulty of training RNNs which in the past had led to the near abandonment in field of RNN research [20].

$\| \nabla_{\mathrm{w}} \mathrm{f}(\mathrm{x})_{\mathrm{n}}^{\mathrm{m}}=\sum_{\mathrm{w}_{\mathrm{H}_{\mathrm{n}} \mathrm{I} \mathrm{W}}}\left|\mathrm{w}_{\mathrm{H}}^{\mathrm{N}} \mathrm{y}_{\mathrm{H}}\right|^{\mathrm{m}}$

Training MLPs for image denoising the idea is to learn an MLP that maps noisy image patches onto clean image patches where the noise is reduced or even removed. The parameters of the MLP are estimated by training on pairs of noisy and clean image patches using stochastic gradient descent (Fig. 4). More precisely, we randomly pick a clean patch $\mathrm{y}$ from an image dataset and generate a corresponding noisy patch $\mathrm{x}$ by corrupting y with noise, for instance with additive white Gaussian noise. The MLP parameters are then updated by the back propagation algorithm minimizing the quadratic error between the mapped noisy patch $f(x)$ and the clean patch $y$, i.e. minimizing $(f(x)-y)^{2}$.

To make RNN more efficient, we apply few neural network tricks, described below:

1. Data normalization: The pixel values are transformed to have approximately mean zero and variance close to one. Precisely, presumptuous pixel values between 0 and 1 , we subtract $0: 5$ and divide by $0: 2$.

2. Weight initialization: The weights are sampled from a normal distribution with mean 0 and standard deviation, $\sigma=\sqrt{\mathrm{N}}$, where $\mathrm{N}$ is the number of input units of the corresponding layer. Combined with the first trick, this ensures that both the linear and the non-linear parts of the sigmoid function are reached.

3. Learning rate division: Within each of the several layers, we have performed division operation between the learning rate by $\mathrm{N}$ and the sum of input units of that layer. This made us easy to manipulate the sum of hidden units without modifying the learning rate. The basic learning rate was set to $0: 1$ for all experiments. No regularization was applied on the weights.

\section{CONCLUSION}

We have introduced a new DPCA algorithm with RNN for MSI denoising. Following the unconventional image processing paradigm, the algorithm proves spatially adaptive for vivid spectral bands and is successful in eliminating the micro components of the spectral bands interfering as noisy components, which was earlier a quite daunting task (Fig. 5). For similar blocks and grouping manifested in the study is robust and provides an error rectification of the range of $85-95 \%$ directly from the noisy image; not only it provides sparsity of corollary of homogenous blocks but also gives show superiority over other methods using cited in the literature. We hoped that our results and the scope of the study will find its application in remote sensing of digital imaging in scenarios like that of astronomy photography.

\section{REFERENCES}

1. Cagnazzo M, Poggi G, Verdoliva L. Region-based transform coding of multispectral images. IEEE Trans Image Process 2007;16:2916-26.

2. Dabov K, Foi A, Katkovnik V, Egiazarian K. Color image denoising via sparse 3D collaborative filtering with grouping constraint in luminancechrominance space. In: Proceedings IEEE International Conference on Image Processing. Vol. 1. September; 2007. p. 313-6.

3. Dabov K, Foi A, Katkovnik V, Egiazarian K. Image denoising by sparse 3-D transform-domain collaborative filtering. IEEE Trans Image Process 2007; 16:2080-95.

4. Epstein B, Hingorani R, Shapiro J, Czigler M. Multispectral KLTwavelet data compression for Landsat thematic mapper images. In: Data Compression Conference, 1992. DCC '92, March; 1992. p. 200-8.

5. Finlayson G, Hordley S, Morovic P. Using the spectra cube to build a multispectral image database. In: Proceedings Second European Conference on Color in Graphics, Imaging and Vision, CGIV 2004, Aachen, Germany, April; 2004. p. 268-74.

6. Foi A. Clipped noisy images: Heteroskedastic modeling and practical denoising. Signal Processing 2009;89(12):2609-29.

7. Foi A, Trimeche M, Katkovnik V, Egiazarian K. Practical poissoniangaussian noise modeling and fitting for single-image raw-data. IEEE Trans Image Process 2008;17(10):1737-54.

8. Hordley S, Finalyson G, Morovic P. A multi-spectral image database and its application to image rendering across illumination. In: Image and Graphics, Proceedings Third International Conference on, December; 2004. p. 394-7.

9. Katkovnik V, Foi A, Egiazarian K, Astola J. From local kernel to nonlocal multiple-model image denoising. Int J Comput Vis 2010;86:1-32.

10. Lansel S. Denoise Lab. Available from: http://www.stanford. edu/ $\sim$ slansel/DenoiseLab.

11. Saghri JA, Tescher AG. Near-lossless bandwidth compression for radiometric data. Opt Eng 1991;30:934-9.

12. Tretter D, Bouman CA. Optimal transforms for multispectral and multilayer image coding. IEEE Trans Image Process 1995;4:296-308.

13. Vansteenkiste E, Van der Weken D, Philips W, Kerre E. Perceived image quality measurement of state-of-the-art noise reduction schemes. In: Lecture Notes in Computer Science ACIVS. Vol. 4179. Antwerp, Belgium: Springer; 2006. p. 114-24.

14. Mahoney M. Text compression as a test for artificial intelligence. In AAAI/IAAI; 1999. p. 486-502.

15. Joshua TG. A bit of progress in language modeling: Extended version. In: Technical Report MSR-TR-2001-72. Redmond, WA: Microsoft Research; 2001.

16. Bengio Y, Ducharme R, Vincent P. A neural probabilistic language model. J Mach Learn Res 2003;3:1137-55.

17. Schwenk H, Gauvain JL. Training neural network language models on very large corpora. In: Proceedings Joint Conference HLT/EMNLP, October; 2005

18. Rai A. A Novel decomposable pixel component analysis algorithm for automating multispectral satellite image denoising. Res Rev J Embed Syst Appl 2015;2(3):18-25.

19. Rai A. Multispectral image denoising using Bi-directional recurrent neural network with DPCA algorithm. J Image Process Pattern Recognit Prog 2015;2(1):25-30

20. Rai A. An introduction of smart self-learning shell programming interface. J Adv Shell Program 2015;1(2):3-6. 